\title{
Heterosis and inbreeding depression in relation to heterotic parameters in bread wheat (Triticum aestivum L.) under late sown condition
}

Jaydev Kumar ${ }^{1 *}$, Arun Kumar ${ }^{2}$, Mukul Kumar ${ }^{3}$, Sanjay Kumar Singh ${ }^{1}$, Lokendra Singh ${ }^{1}$ and Gyanendra Pratap Singh ${ }^{4}$

${ }^{1}$ Chandra Shekhar Azad University of Agriculture and Technology, Kanpur, Uttar Pradesh, India

${ }^{2}$ Govind Ballabh Pant University of Agriculture and Technology, Pantnagar, Uttarakhand, India

${ }^{3}$ Mandan Bharti Agriculture College, Saharsa, Bihar, India

${ }^{4} I C A R-I n d i a n$ Institute of Wheat and Barley Research, Karnal, Haryana, India

\section{Article history}

Received: 18-02-2017

Revised : 29-03-2017

Accepted: 13-06-2017

\section{Citation}

Kumar J, A Kumar, M Kumar, SK Singh, L Singh and GP Singh. 2017. Heterosis and inbreeding depression in relation to heterotic parameters in bread wheat (Triticum aestivum L.) under late sown condition. Journal of Wheat Research 9(1): 32-41.

doi.org/10.25174/2249-

4065/2017/67482

\section{*Corresponding author}

Email: jaydev.140@rediffmail.com

(C) Society for Advancement of Wheat and Barley Research

\begin{abstract}
The present investigation on heterosis and inbreeding depression was conducted on genetically diverse 10 parent diallel crosses of bread wheat. The experimental materials comprised 100 genotypes including 10 parents and their $F_{1} s$ and $F_{2} s$ progenies and trial was conducted in randomized complete block deign with three replication at Economic Botanical Research Farm, Nawabganj of C.S. Azad University of Agriculture and Technology, Kanpur, Uttar Pradesh, India, during 2013-14 and 2014-15. The analysis of variance revealed that the all components of variances showed significant differences for almost the traits under studied over both generations. The result of economic heterosis revealed that the cross combinations, DBW 14/K 0424, K 9162/K 9423, K 9533/K 0307, K 1114/K 0424 and $\mathrm{K}$ 1114/NW 2036 were good for grain yield per plant along with range of $\mathbf{- 8 8 . 5 4}$ (K 0424/K 0911) to 28.52 per cent (DBW 14/K 0424) and other traits compared to $45 \mathrm{~F}_{1} \mathrm{~s}$ whereas, all these cross combinations also had significant grain yield demission in $\mathrm{F}_{2} \mathrm{~s}$ results of increase of homozygosity among the crosses in succeeding generations. The range of inbreeding depression varied from50.36 (K 1114/K 9423) to 49.12 percent (K 0911/K 0307) in $\mathrm{F}_{2} \mathrm{~s}$. Accordingly, these cross combinations may be utilized for improving grain yield as well as production of better transgressive segregants in advance generations for maintain of specific gene pool of bread wheat through breeding program in future.
\end{abstract}

Key words: Diallel, heterosis, inbreeding depression and grain yield

\section{Introduction}

Among cereals, wheat possesses second rank after rice and is the staple food especially in Northern part of the country, where the people are mostly vegetarian. The crop is grown successfully between the altitude of 300 and 600 north and 270 and 400 south globally. Wheat is extensively cultivated under diverse agro-climatic conditions in India covering most of the states, except Kerala. Last few decades, wheat production is decreased due to abiotic stress like high temperature at flowering time of crop (Kumar et al., 2016). To overcome of abiotic stress under wheat cultivation is possible to develop specific gene pool as well as high yielding cultivars against rising temperature through heterosis breeding programme. Because the magnitude of heterosis help in determining genetic diversity and serves as a guide in the selection of desirable parents. Simultaneous studies of heterosis and inbreeding depression estimates give an idea about gene action involved and help in devising breeding methodology for further improvement (Deshpandey and Nayeem, 1999). Inbreeding is basic mechanism for providing the base material for selection because it produced better segregants in wheat crop (Gaur, 2014 and Kumar et al., 2016). Knowledge on the expression levels of the heterosis 
and inbreeding depression are useful to help breeders choose the best hybrid combinations which will serve as the basis for the selection of superior genotypes. Thus, the present investigation was carried out to study the heterosis in $\mathrm{F}_{1}$ over economic parent and inbreeding depression over $\mathrm{F}_{2}$ generations for grain yield and its related traits in wheat under late sown condition.

\section{Materials and methods}

The present investigation was carried out at Economic Botanical Research Farm, Chandra Shekhar Azad University of Agriculture and Technology, Kanpur, Uttar Pradesh during Rabi2014-15. Ten diverse wheat genotypes namely K 9533, K 9162, K 1114, DBW 14, K 0607, K 0424, K 0911, K 0307, NW 2036 and K 9423 were selected as parents on the basis of their vast diversity. Crosses were attempted during Rabi, 2013-14 to generate $\mathrm{F}_{1}$ 's and $\mathrm{F}_{1}$ 's were advanced into $\mathrm{F}_{2}$ 's at ICAR-Indian Institute of Wheat and Barley Research, Regional Station, Dalang Maidan, Lahul Spiti (H.P.) during off season in year of 2014.Final experimental material comprising 10 parents along with their $45 \mathrm{~F}_{1}$ 's and $45 \mathrm{~F}_{2}$ 's were evaluated during Rabi, 2014-15 in randomized block design with three replications. Sowing was done by manually the seeds at a distance of $5 \mathrm{~cm}$ plant to plant in the rows of $4 \mathrm{~m}$ length with row to row spacing of 20 centimeter.During crop period, all recommended agronomic practices were adopted to raise the good crop. The observations were recorded from the five competitive plants in parents and theirF1s and ten plant from their F2s progenies, taken at randomly for the following traits viz.,number of effective tillers per plant, number of spikelets per spike, number of grains per spike, grain weight per spike (g), spike length $(\mathrm{cm})$, biological yield per plant (g), harvest index (\%), 1000-seed weight (g), spike density, protein content (\%) and grain yield per plant (g). Analysis of variance, heterosis and inbreeding depression were calculated as per standard procedures (Fonseca and Patterson, 1968).

\section{Result and discussion}

The analysis of variance of treatments was computed for all the eleven characters and their mean sum of squares given in Table1 (a) and Table 1 (b). Based on Table1 (a) and Table 1 (b), analysis of variances among eleven characters in a 10 parents diallel crosses (excluding reciprocal cross) expressed highly vast of an appreciable amount of variability in the base material as well as in the material.Heterosis was calculated in per cent over economic parent for all eleven characters. Heterosis and inbreeding depression is given in Table 2.
The widely adopted and released variety of K 0307 was considered as economic parent for the estimation of economic heterosis for all traits under studied and inbreeding depression of $\mathrm{F}_{2}$ generation has also been studied (Meredith and Bridge, 1972 and Singh and Narayanam, 2009).The nature and magnitude of heterosis, some crosses exhibited significant and positive heterosis varied from character to character. The range of economic heterosis for number of effective tillers per plant varied from -33.24 in K 1114/K 9423 to 43.38 in $\mathrm{K} 1114 / \mathrm{K} 0911$. Out of 45 cross combinations, only eighteen cross combinations showed positive and significant heterosis. Top two cross combinations namely, $\mathrm{K}$ 1114/K 0911 and K 1114/NW 2036 expressed more number of effective tillers per plant whereas, out of 45 , only four cross combination had no inbreeding depression for the concern trait which can be used for maintain the specific gene pool for further utilization of improvement of wheat. For number of spikelets per spike, five cross combination displayed significant economic heterosis which ranged from -29.43 to 9.39 . K 1114/K 424 expressed high number of spikelets indicates significant economic heterosis whereas, same cross reflect high positive significant value of inbreeding depression for number of spikelets per spike. Similar results were also reported by Singh, (2003) and Jahanzeb and Khaliq, (2004). Economic heterosis for number of grains per spike ranged from -48.63 to 13.29. Cross combinations, K 1114/K 0607 exhibited desirable number grains per spike whereas, eleven crosses out of 45, express negative and significant inbreeding depression. More desirable cross combination K 1114/K 424 showed negative and significant inbreeding depression for the number of grains per spike. The range of economic heterosis for grain weight per spike varied from -96.15 to 12.67. Only five cross combinations showed positive and significant heterosis. K 307/K 9423 had high weight of grain per spike. 12 cross combinations had no inbreeding depression and range of inbreeding depression varied from -29.23 to 33.21 . The similar findings were reported by Nawracala et al., (2006) and Singh et al., (2008). In case of spike length, none of cross combination had significant value of heterosis which is ranged from -31.07 to -3.29 whereas, range of inbreeding depression varied from -14.65 to 16.98 . Top two cross combination were K 1114/K 0607 and K 9162/K 1114 for inbreeding depression expressed more desirable transgressive segregants. For biological yield per plant, eighteen cross combinations exhibited positive and significant economic heterosis which ranged from -48.12 to 27.13. DBW 14/K 424 and K 0911/K 0307 had more biological yield. 


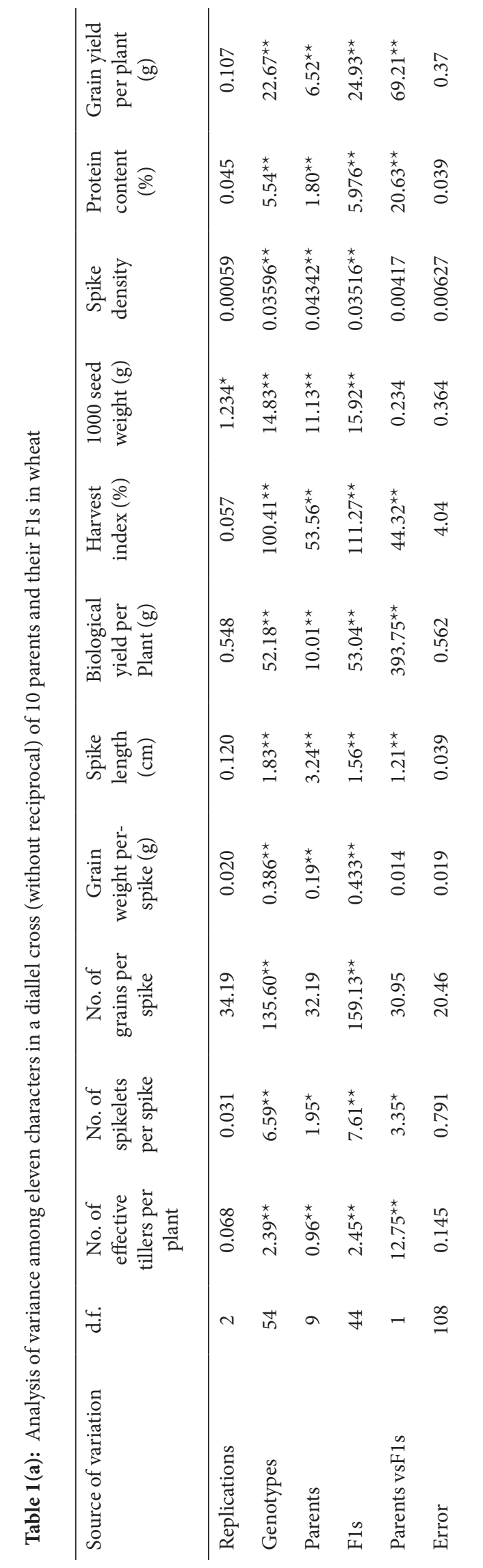

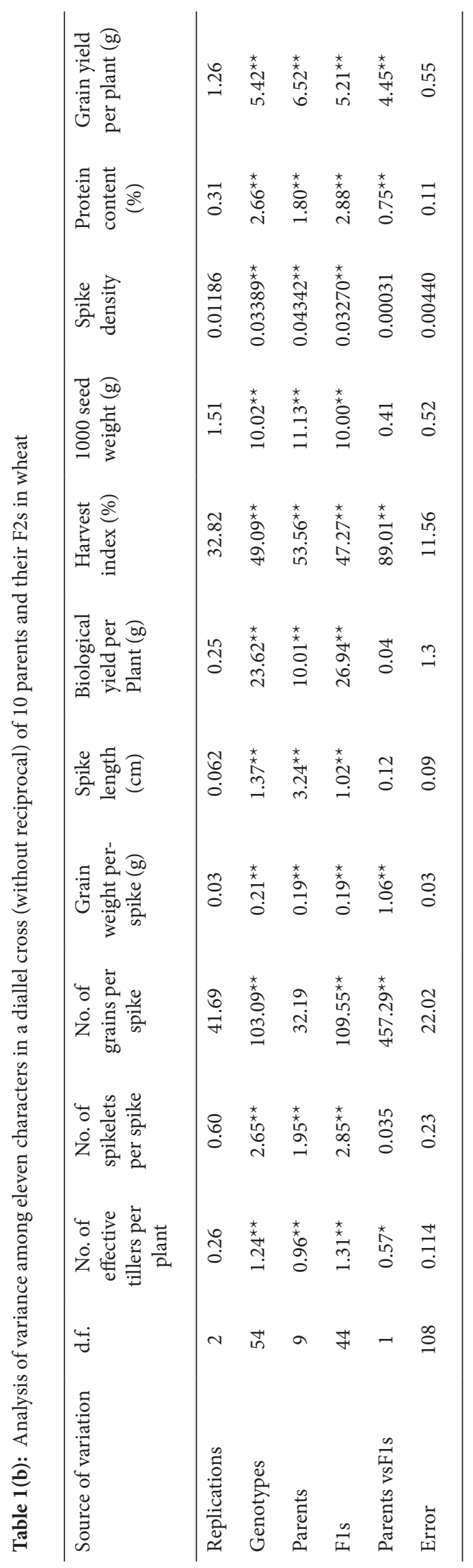


Table 2. Estimates of heterosis over economic parent (per cent) and inbreeding depression (per cent) in a 10 parent diallel crosses fordefferent characters in bread wheat

\begin{tabular}{|c|c|c|c|c|c|c|}
\hline \multirow[t]{2}{*}{ Crosses } & \multicolumn{2}{|c|}{$\begin{array}{c}\text { No. of effective tillers per } \\
\text { plant }\end{array}$} & \multicolumn{2}{|c|}{$\begin{array}{l}\text { No. of spikelets per } \\
\text { spike }\end{array}$} & \multicolumn{2}{|c|}{ No. of grains per spike } \\
\hline & Heterosis & $\begin{array}{l}\text { Inbreeding } \\
\text { depression }\end{array}$ & Heterosis & $\begin{array}{l}\text { Inbreeding } \\
\text { depression }\end{array}$ & Heterosis & $\begin{array}{l}\text { Inbreeding } \\
\text { depression }\end{array}$ \\
\hline 1.K 9533/K 9162 & 9.40 & 4.00 & 3.05 & 6.35 & -4.95 & $21.28^{*}$ \\
\hline 2.K 9533/K 1114 & $15.01^{*}$ & $20.00 * *$ & $7.45^{*}$ & 5.15 & -10.06 & 9.70 \\
\hline 3. K 9533/DBW 14 & -6.09 & 7.81 & -3.51 & 2.03 & $-17.53^{*}$ & $16.45 *$ \\
\hline 4.K 9533/K 0607 & $23.61^{* *}$ & $11.24^{*}$ & 1.17 & 3.56 & -2.46 & 12.75 \\
\hline 5.K 9533/K 0424 & -6.09 & $17.19 *$ & $-29.43^{* *}$ & -25.00 & -14.19 & 6.84 \\
\hline 6.K 9533/K 0911 & 8.11 & -2.70 & 4.28 & 3.76 & -0.68 & 12.53 \\
\hline 7. K 9533/ K 0307 & $24.50^{* *}$ & 11.11 & 3.05 & 4.44 & 7.57 & 7.67 \\
\hline 8. K 9533/NW 2036 & -7.86 & 14.29 & -2.16 & 2.68 & $-17.77 * *$ & 13.16 \\
\hline 9. K 9533/K 9423 & $17.18^{* *}$ & 3.66 & -3.51 & 2.71 & $-14.96^{*}$ & -1.59 \\
\hline 10. K 9162/K 1114 & -4.62 & 9.23 & $-16.14^{* *}$ & $-4.56 *$ & $-28.58^{* *}$ & 11.41 \\
\hline 11.K 9162/DBW 14 & -4.62 & $12.31 *$ & $-19.76^{* *}$ & -2.35 & $-27.08 * *$ & -3.22 \\
\hline 12. K 9162/K 0607 & $12.88^{*}$ & 3.85 & -2.83 & 0.67 & $-17.43^{*}$ & 7.31 \\
\hline 13. K 9162/K 0424 & $12.88^{*}$ & 15.38 & -2.83 & 3.37 & -2.83 & 17.06 \\
\hline 14. K 9162/K 0911 & 8.11 & 6.76 & $-7.16^{* *}$ & -2.11 & $-15.10 *$ & 2.39 \\
\hline 15. K9162/K 0307 & $20.94^{* *}$ & 10.47 & -2.16 & 4.35 & -5.72 & 18.39 \\
\hline 16. K 9162/NW 2036 & $-23.43^{* *}$ & -1.82 & $-8.30^{*}$ & -4.26 & $-35.41^{* *}$ & -12.17 \\
\hline 17. K 9162/K 9423 & $27.75^{* *}$ & $15.96 * *$ & 1.78 & 5.47 & 10.43 & $26.73 *$ \\
\hline 18. K 1114/DBW 14 & -4.62 & 4.62 & -4.25 & $-4.10 *$ & -10.15 & 16.12 \\
\hline 19. K 1114/K 0607 & -2.95 & -13.64 & $-28.86^{* *}$ & $-32.91 * *$ & $13.29^{*}$ & $39.26 * *$ \\
\hline 20. K 1114/K 0424 & $20.11^{* *}$ & $23.53^{* *}$ & $9.39^{* *}$ & $23.74 * *$ & $-48.63^{* *}$ & $-38.87 *$ \\
\hline 21. K 1114/K 0911 & $43.38^{* *}$ & $14.17 * *$ & -2.16 & 0.67 & $-26.14^{* *}$ & 15.12 \\
\hline 22. K 1114/K 0307 & 1.52 & 5.80 & $-8.30 *$ & -3.19 & $-17.60^{*}$ & -2.57 \\
\hline 23. K 1114/NW 2036 & $33.38^{* *}$ & $21.57 * *$ & -5.66 & 0.00 & -12.41 & 13.21 \\
\hline 24. K 1114/K 9423 & $-33.24^{* *}$ & -9.80 & -5.66 & $5.54 *$ & $-36.46^{* *}$ & -1.73 \\
\hline 25. DBW 14/K 0607 & $21.90 * *$ & $13.79 * *$ & $7.75^{*}$ & $9.67^{* *}$ & -5.07 & 4.36 \\
\hline 26. DBW 14/K 0424 & 27.75 & 20.21 & $7.75^{*}$ & $6.04 *$ & 10.43 & 14.86 \\
\hline 27. DBW 14/ K 0911 & -6.09 & 6.25 & -2.83 & 2.02 & $-39.76^{* *}$ & 9.98 \\
\hline 28. DBW 14/K 0307 & 5.62 & 0.00 & 3.05 & 0.32 & -10.84 & 18.39 \\
\hline 29. DBW 14/NW 2036 & -4.62 & -7.69 & 3.05 & 0.63 & $-20.37 * *$ & -3.05 \\
\hline 30. DBW 14/K 9423 & $14.04^{*}$ & $29.11 * *$ & -0.15 & 2.62 & 4.41 & 18.61 \\
\hline 31. K 0607/K 0424 & $14.04^{*}$ & $18.99 *$ & -0.79 & 0.00 & $-18.08^{* *}$ & -7.07 \\
\hline 32. K 0607/K 0911 & 10.65 & $19.74 * *$ & $8.82^{* *}$ & $12.54 * *$ & $11.43^{*}$ & $19.64 *$ \\
\hline 33. K 0607/K 0307 & $21.90^{* *}$ & $34.48 * *$ & -0.15 & $8.20 * *$ & 2.92 & 22.60 \\
\hline 34. K 0607/NW 2036 & 11.70 & 18.18 & $-10.65^{* *}$ & $9.06 *$ & -5.07 & $31.23 * *$ \\
\hline 35. K 0607/K 9423 & 5.62 & $16.67 * *$ & -0.15 & 5.25 & $-31.89 * *$ & 19.00 \\
\hline 36. K 0424/K 0911 & $-23.43^{* *}$ & -21.82 & $-22.65^{* *}$ & $-17.67 * *$ & $-39.52^{* *}$ & -6.91 \\
\hline 37. K 0424/K 0307 & -4.62 & 9.23 & -7.16 & 0.70 & -6.50 & 4.29 \\
\hline 38. K 0424/NW 2036 & $-30.55^{* *}$ & -19.23 & -7.16 & 0.00 & $-19.05^{*}$ & -2.88 \\
\hline 39. K 0424/K 9423 & -9.69 & 4.84 & 1.78 & 4.50 & -10.69 & 7.40 \\
\hline 40. K 0911/K 0307 & $20.11^{* *}$ & $28.24 * *$ & 3.05 & 5.08 & 8.06 & $27.12 *$ \\
\hline 41. K 0911/NW 2036 & $18.08^{* *}$ & $21.69 *$ & 6.05 & 4.92 & 10.06 & 17.31 \\
\hline 42. K 0911/K 9423 & $4.04^{*}$ & 5.06 & -7.16 & -5.61 & -10.42 & -15.39 \\
\hline 43. K 0307/NW 2036 & 8.11 & 6.76 & 2.44 & 1.28 & -3.93 & -18.20 \\
\hline 44. K 0307/K 9423 & 10.65 & 11.84 & 2.12 & $6.41 *$ & $11.53^{*}$ & $24.36 *$ \\
\hline 45. NW 2036/ K 9423 & -7.86 & 17.46 & $-10.23^{* *}$ & 0.72 & -13.74 & $16.25^{*}$ \\
\hline $\mathrm{SE} \pm$ & 0.31 & & & 72 & 3.69 & \\
\hline$+(\operatorname{sig})$ & $11(18)$ & $22(16)$ & $12(5)$ & $27(8)$ & $7(3)$ & $24(9)$ \\
\hline$-(\operatorname{sig})$ & $12(4)$ & $7(0)$ & $18(10)$ & $6(4)$ & $17(18)$ & $11(1)$ \\
\hline
\end{tabular}


Table 2. Continued

\begin{tabular}{|c|c|c|c|c|c|c|}
\hline \multirow[t]{2}{*}{ Crosses } & \multicolumn{2}{|c|}{$\begin{array}{c}\text { Grains weight per } \\
\text { spike }(\mathrm{g})\end{array}$} & \multicolumn{2}{|c|}{$\begin{array}{l}\text { Spike length } \\
(\mathrm{cm})\end{array}$} & \multicolumn{2}{|c|}{$\begin{array}{c}\text { Biological yield per } \\
\text { plant }(\mathrm{g})\end{array}$} \\
\hline & Heterosis & $\begin{array}{l}\text { Inbreeding } \\
\text { depression }\end{array}$ & Heterosis & $\begin{array}{l}\text { Inbreeding } \\
\text { depression }\end{array}$ & Heterosis & $\begin{array}{l}\text { Inbreeding } \\
\text { depression }\end{array}$ \\
\hline 1.K 9533/K 9162 & $-14.86^{* *}$ & $22.82 * *$ & $-14.12^{* *}$ & 1.97 & $6.19^{*}$ & $15.28 * *$ \\
\hline 2.K 9533/K 1114 & $-20.28^{* *}$ & $23.27 *$ & $-6.55^{* *}$ & $5.53 *$ & $14.05^{* *}$ & $31.30 * *$ \\
\hline 3. K 9533/DBW 14 & $-32.81^{* *}$ & $13.57 * *$ & $-21.29^{* *}$ & $-9.88 * *$ & $-25.37 * *$ & $9.56 *$ \\
\hline 4.K 9533/K 0607 & -9.44 & $21.29 * *$ & $-10.93^{* *}$ & $14.79 * *$ & $17.83^{* *}$ & $16.61 *$ \\
\hline 5.K 9533/K 0424 & $-28.79^{* *}$ & 11.60 & $-20.21 * *$ & $-6.82 *$ & $-11.60^{* *}$ & $21.75 *$ \\
\hline 6.K 9533/K 0911 & $-10.39^{*}$ & 14.57 & $-11.29^{* *}$ & $13.46 * *$ & $5.85^{*}$ & 19.18 \\
\hline 7. K 9533/ K 0307 & 5.20 & $15.59 *$ & $-3.61^{* *}$ & $6.65 *$ & $20.70^{* *}$ & 2.17 \\
\hline 8. K 9533/NW 2036 & $-35.64^{* *}$ & 8.67 & $-11.29 * *$ & 3.57 & $-6.43^{*}$ & $12.40 * *$ \\
\hline 9. K 9533/K 9423 & $-30.10 * *$ & -0.34 & $-20.86^{* *}$ & $7.16^{*}$ & -5.41 & -10.47 \\
\hline 10. K 9162/K 1114 & $-39.34^{* *}$ & 4.37 & $-31.07 * *$ & $-11.00 *$ & $-12.03^{*}$ & $18.35 * *$ \\
\hline 11.K 9162/DBW 14 & $-45.71^{* *}$ & -2.86 & $-19.47 * *$ & $-3.54 *$ & $-23.56^{* *}$ & 0.98 \\
\hline 12. K 9162/K 0607 & $-21.43 * *$ & 5.25 & $-9.49 * *$ & 2.70 & $-5.89 *$ & 5.73 \\
\hline 13. K 9162/K 0424 & $-13.84^{* *}$ & 14.88 & $-14.41^{* *}$ & $5.08 *$ & -2.34 & $14.22 *$ \\
\hline 14. K 9162/K 0911 & $-26.87 * *$ & 1.16 & $-20.21^{* *}$ & -2.67 & -0.87 & 2.09 \\
\hline 15. K9162/K 0307 & -2.41 & $26.94 * *$ & $-3.29 * *$ & $2.81 *$ & $18.47^{* *}$ & $23.56 * *$ \\
\hline 16. K 9162/NW 2036 & $-47.40^{* *}$ & -20.66 & $-27.00 * *$ & -3.13 & -1.27 & $24.89 * *$ \\
\hline 17. K 9162/K 9423 & $10.84^{* *}$ & $29.75 *$ & $-10.38^{* *}$ & $2.45 *$ & $22.21^{* *}$ & $15.22 * *$ \\
\hline 18. K 1114/DBW 14 & $-22.01^{* *}$ & 11.34 & $-12.22^{* *}$ & 4.16 & $-18.33^{* *}$ & 8.37 \\
\hline 19. K 1114/K 0607 & $-75.86^{* *}$ & -17.74 & $-28.94^{* *}$ & $-14.65^{* *}$ & $-48.12^{* *}$ & -9.37 \\
\hline 20. K 1114/K 0424 & $8.27^{*}$ & $31.06 * *$ & $-9.14^{* *}$ & $16.98^{* *}$ & $18.55^{* *}$ & $36.59 * *$ \\
\hline 21. K 1114/K 0911 & $-47.40^{* *}$ & 11.20 & $-22.39 * *$ & -0.60 & $19.29 * *$ & $29.54 * *$ \\
\hline 22. K 1114/K 0307 & $-26.87 * *$ & -5.48 & $-20.54^{* *}$ & 0.00 & 2.44 & $22.45^{* *}$ \\
\hline 23. K 1114/NW 2036 & -8.05 & $16.10 *$ & $-20.54^{* *}$ & 1.49 & $22.99^{* *}$ & $26.08 * *$ \\
\hline 24. K 1114/K 9423 & $-52.69^{* *}$ & -7.39 & $-13.73^{* *}$ & $7.58 * *$ & $-17.49^{* *}$ & $14.48^{* *}$ \\
\hline 25. DBW 14/K 0607 & $-23.79^{* *}$ & 1.94 & $-5.72 * *$ & $6.53 *$ & 5.89 & $14.50 * *$ \\
\hline 26. DBW 14/K 0424 & $12.07^{* *}$ & $15.07 * *$ & $-14.70^{* *}$ & 1.42 & $27.13^{* *}$ & $28.69 * *$ \\
\hline 27. DBW 14/ K 0911 & $-49.12 * *$ & $14.79 *$ & $-23.06^{* *}$ & 0.30 & $-10.28^{*}$ & $18.59 * *$ \\
\hline 28. DBW 14/K 0307 & $-30.10^{* *}$ & 7.50 & $-6.55^{* *}$ & $4.47 * *$ & -4.46 & $26.37 * *$ \\
\hline 29. DBW 14/NW 2036 & $-27.50^{* *}$ & -5.83 & $-23.85^{* *}$ & -1.53 & $-19.92^{* *}$ & -12.67 \\
\hline 30. DBW 14/K 9423 & $-13.84^{* *}$ & $14.71 *$ & $-15.09^{* *}$ & 3.69 & $-9.96^{* *}$ & $15.53 * *$ \\
\hline 31. K 0607/K 0424 & $-32.81^{* *}$ & $-17.22 *$ & $-13.16^{* *}$ & 0.00 & 1.99 & $8.53 * *$ \\
\hline 32. K 0607/K 0911 & $12.07 * *$ & $23.13 * *$ & $-4.41^{* *}$ & $6.70 * *$ & $23.37 * *$ & $31.17 * *$ \\
\hline 33. K 0607/K 0307 & -7.14 & $20.42 *$ & $-6.55^{* *}$ & $8.42 *$ & $18.15^{* *}$ & $41.00 * *$ \\
\hline 34. K 0607/NW 2036 & $-25.00 * *$ & $26.02 * *$ & $-8.26^{* *}$ & $4.28 *$ & $6.91^{* *}$ & $41.41^{* *}$ \\
\hline 35. K 0607/K 9423 & $-35.64^{* *}$ & $24.11 *$ & $-9.14^{* *}$ & $7.01 *$ & $-11.82^{* *}$ & $16.09 *$ \\
\hline 36. K 0424/K 0911 & $-96.15^{* *}$ & $-29.23 *$ & $-17.39^{* *}$ & 4.06 & $-38.31^{* *}$ & 0.12 \\
\hline 37. K 0424/K 0307 & $-11.35^{*}$ & 6.26 & $-22.73^{* *}$ & -3.33 & -3.30 & $14.79 *$ \\
\hline 38. K 0424/NW 2036 & $-16.97^{* *}$ & -1.84 & $-22.39 * *$ & -1.81 & $-30.30 * *$ & $-7.98 *$ \\
\hline 39. K 0424/K 9423 & $-13.33^{* *}$ & 12.76 & $-7.14^{* *}$ & 2.65 & $-29.42^{* *}$ & 4.23 \\
\hline 40. K 0911/K 0307 & 6.25 & $33.21 * *$ & $-14.41^{* *}$ & -1.41 & $24.17^{* *}$ & $40.53 * *$ \\
\hline 41. K 0911/NW 2036 & -4.94 & 7.68 & $-7.74^{* *}$ & $5.32 *$ & $11.22^{* *}$ & $15.55 * *$ \\
\hline 42. K 0911/K 9423 & $-23.79 * *$ & -13.59 & $-12.78^{* *}$ & 0.28 & $5.77^{*}$ & -3.89 \\
\hline 43. K 0307/NW 2036 & $-15.38^{* *}$ & -13.73 & $-10.02^{* *}$ & 0.82 & 3.50 & 1.19 \\
\hline 44. K 0307/K 9423 & $12.67^{* *}$ & $32.95 *$ & $-12.22^{* *}$ & 0.83 & $15.59^{* *}$ & $27.62 * *$ \\
\hline 45. NW 2036/ K 9423 & $-23.79^{* *}$ & 13.57 & $-21.95^{* *}$ & 4.52 & $-23.49^{* *}$ & $13.14 * *$ \\
\hline $\mathrm{SE} \pm$ & \multicolumn{2}{|c|}{0.11} & \multicolumn{2}{|c|}{0.16} & \multicolumn{2}{|c|}{0.61} \\
\hline$+(\operatorname{sig})$ & $2(5)$ & $15(18)$ & $0(0)$ & $16(17)$ & $4(18)$ & $9(31)$ \\
\hline$-(\operatorname{sig})$ & $5(33)$ & $10(2)$ & $0(45)$ & $7(5)$ & $6(17)$ & $4(1)$ \\
\hline
\end{tabular}


Table 2. Continued

\begin{tabular}{|c|c|c|c|c|c|c|}
\hline \multirow[t]{2}{*}{ Crosses } & \multicolumn{2}{|c|}{$\begin{array}{c}\text { Harvest index } \\
(\%)\end{array}$} & \multicolumn{2}{|c|}{$\begin{array}{c}1000 \text { seed weight } \\
(\mathrm{g})\end{array}$} & \multicolumn{2}{|c|}{$\begin{array}{c}\text { Spike } \\
\text { density }\end{array}$} \\
\hline & Heterosis & $\begin{array}{l}\text { Inbreeding } \\
\text { depression }\end{array}$ & Heterosis & $\begin{array}{l}\text { Inbreeding } \\
\text { depression }\end{array}$ & Heterosis & $\begin{array}{l}\text { Inbreeding } \\
\text { depression }\end{array}$ \\
\hline 1.K 9533/K 9162 & $-14.61^{* *}$ & 12.02 & $-11.25^{* *}$ & 0.80 & $14.69^{* *}$ & 4.32 \\
\hline 2.K 9533/K 1114 & $-28.14^{* *}$ & $-7.45 *$ & $-10.48^{* *}$ & 1.01 & $13.22^{* *}$ & -0.57 \\
\hline 3. K 9533/DBW 14 & $-13.35^{* *}$ & 1.19 & $-13.07 * *$ & -5.56 & $14.69^{* *}$ & $10.94^{* *}$ \\
\hline 4.K 9533/K 0607 & $-13.04^{* *}$ & 9.51 & $-10.20^{* *}$ & 1.91 & $10.65^{*}$ & -13.38 \\
\hline 5.K 9533/K 0424 & $-26.20^{* *}$ & -9.74 & $-15.96^{* *}$ & -1.31 & -8.63 & -17.46 \\
\hline 6.K 9533/K 0911 & $-7.68^{*}$ & -4.59 & $-8.99 * *$ & -3.68 & $13.71^{*}$ & -11.40 \\
\hline 7. K 9533/ K 0307 & 2.79 & $23.15^{* *}$ & $-2.40^{*}$ & 3.23 & 6.21 & -2.27 \\
\hline 8. K 9533/NW 2036 & $-38.44^{* *}$ & -10.62 & $-13.33^{* *}$ & $-4.28 *$ & $7.93^{*}$ & -1.01 \\
\hline 9. К 9533/K 9423 & $-11.88^{* *}$ & $10.23 *$ & $-11.87^{* *}$ & 1.71 & $14.20^{* *}$ & -4.92 \\
\hline 10. K 9162/K 1114 & $-27.21^{* *}$ & -7.35 & $-10.07^{* *}$ & -2.49 & $11.70^{* *}$ & 5.85 \\
\hline 11.K 9162/DBW 14 & $-24.53^{* *}$ & -3.02 & $-14.11^{* *}$ & -3.95 & -0.67 & 1.10 \\
\hline 12. K 9162/K 0607 & -4.27 & 2.90 & -0.77 & -0.09 & 6.21 & -2.07 \\
\hline 13. K 9162/K 0424 & -0.29 & 12.91 & $-10.67^{* *}$ & $-2.20 *$ & $10.12^{\text {** }}$ & -1.58 \\
\hline 14. K 9162/K 0911 & $-13.97^{* *}$ & $12.91 *$ & $-6.99^{* *}$ & -1.12 & $11.18^{* *}$ & 0.78 \\
\hline 15. K9162/K 0307 & -2.14 & $14.69 * *$ & -0.23 & 3.61 & 1.31 & 1.52 \\
\hline 16. K 9162/NW 2036 & $-110.28^{* *}$ & $-91.37 * *$ & $-6.96^{* *}$ & -0.39 & $14.69^{* *}$ & -0.94 \\
\hline 17. K $9162 / \mathrm{K} 9423$ & 5.02 & $22.99 * *$ & -0.93 & -0.05 & $10.65^{* *}$ & 2.95 \\
\hline 18. K 1114/DBW 14 & $-15.29^{* *}$ & 7.26 & $-10.26^{* *}$ & 0.32 & 6.79 & $-8.62 * *$ \\
\hline 19. K 1114/K 0607 & $-23.73^{* *}$ & -7.84 & $-12.21^{* *}$ & -6.45 & 0.00 & $-15.89 * *$ \\
\hline 20. K 1114/K 0424 & 5.25 & $17.51 * *$ & $-4.08^{* *}$ & 13.36 ** & $16.57^{* *}$ & $7.90 *$ \\
\hline 21. K 1114/K 0911 & $-11.08^{* *}$ & -9.65 & $-10.07^{* *}$ & 0.97 & $16.57^{* *}$ & 0.92 \\
\hline 22. K 1114/K 0307 & $-24.35^{\text {** }}$ & -17.79 & -0.75 & $5.01 *$ & $10.12^{* *}$ & -6.34 \\
\hline 23. K 1114/NW 2036 & -1.88 & $11.14^{*}$ & $3.29^{* *}$ & $3.89 *$ & $12.21^{\text {** }}$ & -0.19 \\
\hline 24. K 1114/K 9423 & $-60.07 * *$ & $-75.63 * *$ & $-10.26^{* *}$ & $3.06 * *$ & 6.79 & -2.25 \\
\hline 25. DBW 14/K 0607 & -5.31 & 1.74 & $-13.47^{* *}$ & $-3.62 * *$ & $12.72^{* *}$ & 3.08 \\
\hline 26. DBW $14 /$ K 0424 & 1.90 & 7.58 & 0.02 & $3.25 * *$ & $19.68^{* *}$ & 4.44 \\
\hline 27. DBW 14/ K 0911 & $-40.92^{* *}$ & $-53.89 * *$ & $-4.69^{* *}$ & $7.51 * *$ & $16.11^{* *}$ & 1.47 \\
\hline 28. DBW 14/K 0307 & $-11.22^{* *}$ & -6.69 & $-17.84^{* *}$ & $-14.59 * *$ & $9.04^{*}$ & -4.62 \\
\hline 29. DBW 14/NW 2036 & $-12.38^{* *}$ & -0.27 & $-3.55^{* *}$ & -0.70 & $21.76^{* *}$ & 1.90 \\
\hline 30. DBW 14/K 9423 & $7.38^{*}$ & $26.82 * *$ & $-14.91^{* *}$ & $-5.94 * *$ & $13.22^{* *}$ & -0.96 \\
\hline 31. K 0607/K 0424 & $-15.47^{* *}$ & $-5.30 * *$ & $-7.02^{* *}$ & -2.80 & $10.65^{* *}$ & $-4.13 * *$ \\
\hline 32. K 0607/K 0911 & $-12.67^{* *}$ & 6.50 & 1.18 & $3.12 *$ & $12.72 * *$ & $6.17 *$ \\
\hline 33. K 0607/K 0307 & $-14.12^{* *}$ & -1.93 & -0.68 & $8.55^{* *}$ & 6.21 & -0.41 \\
\hline 34. K 0607/NW 2036 & $-9.59^{* *}$ & -3.00 & $-19.68^{* *}$ & $-8.57 * *$ & -2.03 & 5.19 \\
\hline 35. K 0607/K 9423 & $-8.89^{*}$ & $16.71 *$ & $-10.67^{* *}$ & -1.01 & $8.48^{*}$ & -1.82 \\
\hline 36. K 0424/K 0911 & $-36.64^{* *}$ & $-25.19 *$ & $-17.43^{* *}$ & $-5.44 * *$ & -4.86 & $-22.63 * *$ \\
\hline 37. K 0424/K 0307 & $-13.60^{* *}$ & 1.08 & $-7.09^{* *}$ & $-7.55^{* *}$ & $12.72^{* *}$ & 3.66 \\
\hline 38. K 0424/NW 2036 & $-10.68^{* *}$ & 0.46 & $2.22^{*}$ & -0.76 & $12.21^{* *}$ & 1.74 \\
\hline 39. K 0424/K 9423 & $-5.59^{* *}$ & 7.70 & -2.19 & 0.45 & $8.48^{*}$ & 2.02 \\
\hline 40. K 0911/K 0307 & $-7.38^{*}$ & 14.52 & -0.70 & $7.22 * *$ & $15.17^{* *}$ & $6.36 *$ \\
\hline 41. K 0911/NW 2036 & -6.03 & $10.84^{* *}$ & $-5.41^{*}$ & $-3.59 *$ & $12.72^{* *}$ & -0.38 \\
\hline 42. K 0911/K 9423 & $-9.66^{* *}$ & 5.86 & $-10.40^{* *}$ & $-6.77 * *$ & 5.03 & -6.09 \\
\hline 43. K 0307/NW 2036 & -3.55 & 2.47 & $-7.48^{* *}$ & -0.14 & $11.18^{* *}$ & 0.58 \\
\hline 44. K 0307/K 9423 & 0.89 & $12.42^{* *}$ & 1.47 & $11.82 * *$ & $12.72^{* *}$ & $5.78 *$ \\
\hline 45. NW 2036/ K 9423 & 0.79 & 7.29 & $-5.51^{*}$ & 0.22 & $9.58^{*}$ & -3.58 \\
\hline $\mathrm{SE} \pm$ & \multicolumn{2}{|c|}{1.64} & \multicolumn{2}{|c|}{0.49} & \multicolumn{2}{|c|}{0.064} \\
\hline$+($ sig $)$ & $6(1)$ & $16(11)$ & $3(2)$ & $10(10)$ & $8(33)$ & $16(5)$ \\
\hline -(sig) & $7(31)$ & $12(6)$ & $7(33)$ & $15(10)$ & $4(0)$ & $20(4)$ \\
\hline
\end{tabular}


Table 2. Continued

\begin{tabular}{|c|c|c|c|c|}
\hline \multirow[t]{2}{*}{ Crosses } & \multicolumn{2}{|c|}{$\begin{array}{c}\text { Protein content } \\
(\%)\end{array}$} & \multicolumn{2}{|c|}{ Grain yield per plant $(\mathrm{g})$} \\
\hline & Heterosis & $\begin{array}{l}\text { Inbreeding } \\
\text { depression }\end{array}$ & Heterosis & $\begin{array}{l}\text { Inbreeding } \\
\text { depression }\end{array}$ \\
\hline 1.K 9533/K 9162 & $-6.63^{* *}$ & 1.42 & -7.50 & $25.50 * *$ \\
\hline 2.K 9533/K 1114 & $-4.58 * *$ & -1.11 & $-10.23^{*}$ & 26.12 ** \\
\hline 3. K 9533/DBW 14 & $-18.06^{* *}$ & $-5.33^{*}$ & $-42.12^{* *}$ & $10.70 *$ \\
\hline 4.K 9533/K 0607 & $-23.40^{* *}$ & -3.93 & 7.16 & 24.83 ** \\
\hline 5.K 9533/K 0424 & $-22.20^{* *}$ & 0.00 & $-40.97 * *$ & 14.54 * \\
\hline 6.K 9533/K 0911 & $-15.88^{* *}$ & $7.38 *$ & -1.16 & 16.05 \\
\hline 7. K 9533/ K 0307 & $-17.29 * *$ & $6.85 *$ & $22.91^{* *}$ & $24.83 * *$ \\
\hline 8. K 9533/NW 2036 & $-22.20 * *$ & -8.44 & $-47.28^{* *}$ & 2.86 \\
\hline 9. K 9533/K 9423 & $-11.95^{* *}$ & $11.96 * *$ & $-17.91^{* *}$ & 0.71 \\
\hline 10. K 9162/K 1114 & $-10.38 * *$ & -2.64 & $-42.46^{* *}$ & 12.16 \\
\hline 11.K 9162/DBW 14 & $-10.77^{* *}$ & -4.41 & $-53.79^{* *}$ & -1.09 \\
\hline 12. K 9162/K 0607 & $-7.26^{* *}$ & $6.27 *$ & $-10.43^{*}$ & $9.15 *$ \\
\hline 13. K 9162/K 0424 & $-8.85^{* *}$ & $-9.83 * *$ & -2.53 & $25.59 *$ \\
\hline 14. K 9162/K 0911 & $-4.58^{* *}$ & $9.72 * *$ & $-15.01 * *$ & 14.60 \\
\hline 15. K9162/K 0307 & -2.28 & $5.98 *$ & $16.75^{* *}$ & 34.90 ** \\
\hline 16. K 9162/NW 2036 & $3.46^{* *}$ & $10.26 *$ & $-11.31^{* *}$ & -45.16 \\
\hline 17. K 9162/K 9423 & $-11.75^{* *}$ & 5.64 & $26.14^{* *}$ & $34.69 * *$ \\
\hline 18. K 1114/DBW 14 & $-6.63^{* *}$ & 3.68 & $-36.85^{* *}$ & 14.71 * \\
\hline 19. K 1114/K 0607 & $-22.20^{* *}$ & $-10.06 *$ & $-83.16^{* *}$ & -18.20 \\
\hline 20. K 1114/K 0424 & -0.97 & $8.04 *$ & $22.81^{* *}$ & $47.79 * *$ \\
\hline 21. K 1114/K 0911 & $9.26^{* *}$ & 1.20 & $10.38^{* *}$ & 22.69 ** \\
\hline 22. K 1114/K 0307 & $5.14^{* *}$ & $12.09 * *$ & $-21.19 * *$ & 8.49 \\
\hline 23. K 1114/NW 2036 & $7.92^{* *}$ & $11.74 * *$ & $21.57^{* *}$ & 34.43 ** \\
\hline 24. K 1114/K 9423 & $7.72 * *$ & 0.00 & $-88.25^{* *}$ & $-50.36 *$ \\
\hline 25. DBW 14/K 0607 & $10.55^{* *}$ & $10.45^{* *}$ & 0.90 & $16.14^{* *}$ \\
\hline 26. DBW 14/K 0424 & $13.03^{* *}$ & $15.94^{* *}$ & $28.52^{* *}$ & 34.21 ** \\
\hline 27. DBW 14/ K 0911 & $8.19^{* *}$ & 3.41 & $-55.36^{* *}$ & $-25.21 *$ \\
\hline 28. DBW 14/K 0307 & $-5.20^{* *}$ & 2.23 & $-16.11^{* *}$ & $21.55^{* *}$ \\
\hline 29. DBW 14/NW 2036 & $8.86^{* *}$ & $6.78 * *$ & $-35.03^{* *}$ & -13.22 \\
\hline 30. DBW 14/K 9423 & $12.85^{* *}$ & $18.06 * *$ & -1.84 & $38.18 * *$ \\
\hline 31. K 0607/K 0424 & $5.14^{* *}$ & $5.54 * *$ & $-13.20^{* *}$ & 3.69 \\
\hline 32. K 0607/K 0911 & $16.67^{* *}$ & $17.02 * *$ & $13.68^{* *}$ & 35.62 ** \\
\hline 33. K 0607/K 0307 & $15.72^{* *}$ & 3.49 & 6.52 & 39.80 ** \\
\hline 34. K 0607/NW 2036 & $6.83^{* *}$ & $5.47^{* *}$ & -1.92 & 39.79 ** \\
\hline 35. K 0607/K 9423 & $10.04^{* *}$ & $8.72 * *$ & $-21.80^{* *}$ & $29.97 * *$ \\
\hline 36. K 0424/K 0911 & $11.68^{* *}$ & $12.97 * *$ & $-88.54^{* *}$ & -24.77 \\
\hline 37. K 0424/K 0307 & $9.97 * *$ & $17.50 * *$ & $-17.00^{* *}$ & 15.97 \\
\hline 38. K 0424/NW 2036 & $7.52^{* *}$ & 8.64 ** & $-44.14^{* *}$ & -7.45 \\
\hline 39. K 0424/K 9423 & $13.80^{* *}$ & $14.15^{* *}$ & $-36.55^{* *}$ & 11.70 \\
\hline 40. K 0911/K 0307 & $5.50 * *$ & -1.41 & $18.47^{* *}$ & 49.12 ** \\
\hline 41. K 0911/NW 2036 & $7.92 * *$ & $9.05^{*}$ & 5.87 & 24.68 ** \\
\hline 42. K 0911/K 9423 & $9.45^{* *}$ & $9.79 *$ & -3.05 & 2.43 \\
\hline 43. K 0307/NW 2036 & -1.37 & 0.65 & 0.25 & 3.85 \\
\hline 44. K 0307/K 9423 & $-5.82^{* *}$ & -4.86 & $16.35^{* *}$ & 36.74 ** \\
\hline 45. NW 2036/ K 9423 & $6.41^{* *}$ & $4.80 *$ & $-22.54^{* *}$ & $19.44^{*}$ \\
\hline $\mathrm{SE} \pm$ & \multicolumn{2}{|c|}{0.16} & \multicolumn{2}{|c|}{0.49} \\
\hline$+(\operatorname{sig})$ & $0(23)$ & $10(25)$ & $5(10)$ & $11(26)$ \\
\hline$-(\operatorname{sig})$ & $3(19)$ & $7(3)$ & $6(24)$ & $6(2)$ \\
\hline
\end{tabular}




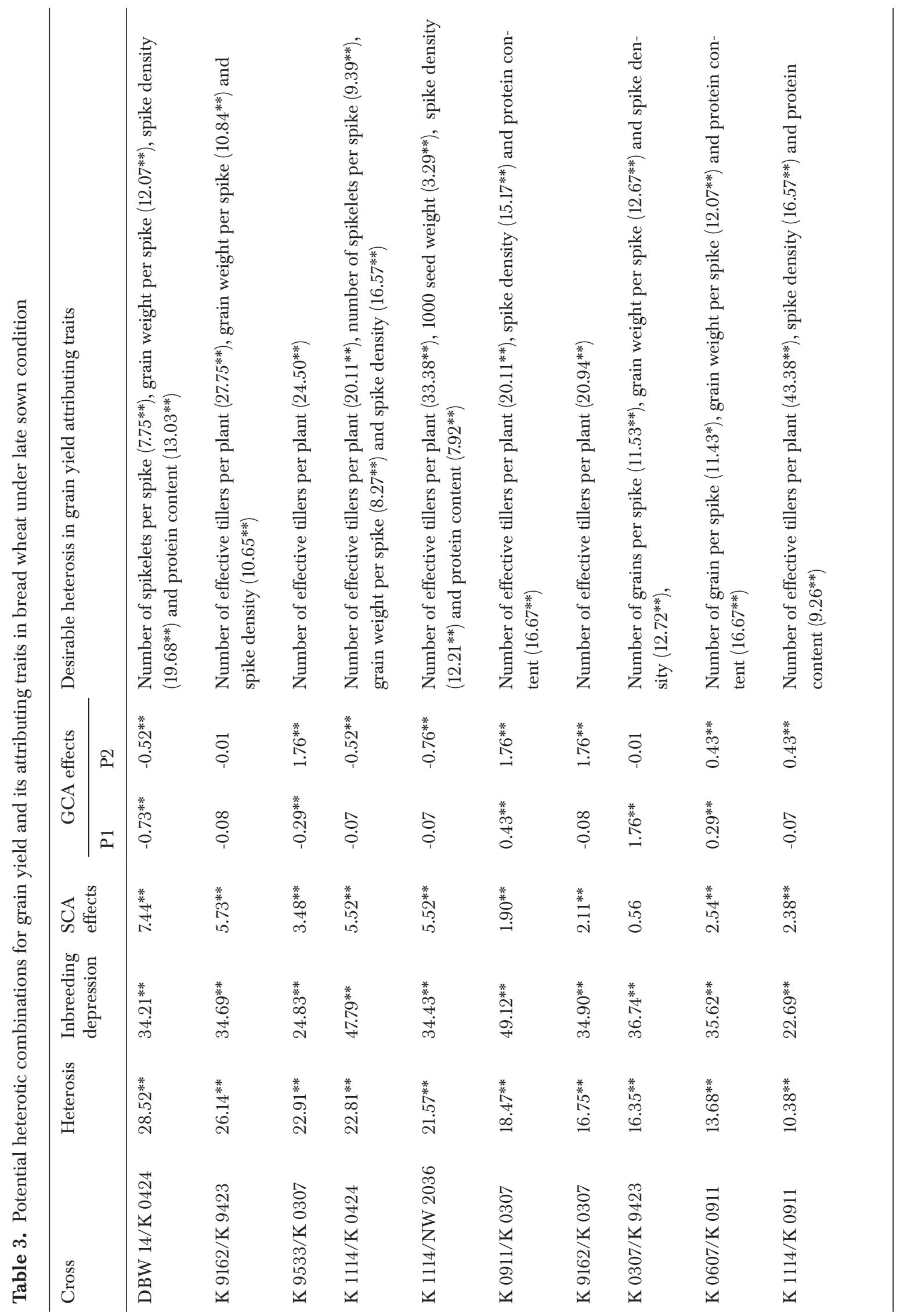


The range of inbreeding depression for biological yield is positioned from -12.67 to 41.41 . Thirty one cross combinations had negative and significant value of inbreeding depression. For development of heat tolerant genotypes in bread wheat, genotypes which have more biological yield should be used further in breeding programme(Desaleet al., 2013 and Kumar et al., 2016). The estimates of economic heterosis for harvest index varied from -110.28 to 7.38 percent. Only one cross combination DBW 14/K 9423 exhibited maximum positive and significant heterosis for harvest index indicated economic importance. These crosses could be of greater value if exploited in breeding programme. Positive heterosis for harvest index was reported by Singh et al. (2013). Whereas, range of inbreeding depression varied from -91.37 to 26.82 for harvest index. Six cross combination out of 45 , expressed desirable inbreeding depression.Crosses K 9162/ NW 2036, K 1114/K 9423, DBW 14/K 0911, K 0424/K 0911 and K 9533/K 1114 were also desirable for straw and showed negative and significant value of inbreeding depression in order to merit.

For 1000 grain weight, desirable amount of positive heterosis was observed and range varied from -19.68 to 3.29. Only two crosses exhibited heterosis in positive and significant direction namely K 1114/NW 2036 and K 0424/NW 2036. Heterosis for 1000-grain weight was earlier reported by Hassan and Saad (1996). In case of range of inbreeding depression of 1000-grain weight varied from -14.59 to 13.36 . Out of 45,25 crosses displayed desirable amount of inbreeding depression. DBW 14/K 0307 and K 0607/NW 2036 were top two crosses expressed super cross in segregating generation. The magnitude of economic heterosis for spike density ranged from -8.63 to 21.76 . Significant positive heterosis was demonstrated by two hybrids, i.e. DBW 14/NW 2036 and DBW 14/K 0424. These crosses could be of high value if used in future breeding strategy. The range of inbreeding depression for spike density varied from -22.63 to 10.93 .24 crosses, out of 45 had negative and significant inbreeding depression indicate concerned trait controlled by additive gene and could be improve though subsequent selection scheme of plant breeding. Top two crosses K 0404/K 0911 and K 9533/K 0424 had more spike density. The magnitude of heterosis for protein content ranged from -23.40 to 16.67 . Out of 45 , twenty three crosses were positive and significant heterosis over economic parent for protein content whereas, nineteen crosses were negative and significant economic heterosis. Crosses K 0607/K 0911, K 0607/K 0307, K 0424/K
9423, DBW 14/K 0424 and DBW 14/K 9423 had positive and significant economic heterosis. Similar results for gluten content were reported by Krystkowiak et al. (2009), Singh and Sharma (2012) and Singh et al. (2014). Ten cross combinations had negative and significant inbreeding depression in protein content. Top two cross K 1114/K 0607 and K 9162/K 0424 showed themselves for more desirable of protein content based on inbreeding depression. The range of heterosis over economic parent for grain yield per plant varied from -88.54 to 28.52. While selecting the plants, grain yield received maximum attention of plant breeder. Therefore, positive heterosis for grain yield is desirable. In case of grain yield per plant, 10 crosses showed significant and positive over economic parent (Table 3). Top five crosses for positive and significant desirable heterosis found in DBW 14/K 0424, K 9162/K 9423, K 9533/K 0307, K 1114/K 0424 and K 1114/ NW 2036. Similar result on positive heterosis for grain yield per plant has been reported by Devi et al. (2013), Desale $e t$ al. (2013) and Singh et al. (2014). Inbreeding depression for grain yield per plant ranged from -50.36 to 49.12 per cent. Only eight cross combination had negative and significant inbreeding depression. First five crosses were K1114/K 9423, K 9162/NW 2036, DBW 14/K 0911, K 0424/K 0911 and K 1114/K 0607.

The linearity among the per cent homozygosity of the parent and the performance indicates that economic increase in grain yield in most of the combinations is due to non additive genetic components, has been cited as a major factor for the manifestation of hybrid vigour. As far as, the heterosis for grain yield per plant is concerned, it was observed that out of 45 , only ten cross combinations showed positive heterosis over economic parent. Accordingly crosses, heterosis respond by high SCA effect along with detonation in their performance in $\mathrm{F}_{2}$ generation for most of crosses (Singh and Narayanam, 2009). Inbreeding depression based on genetic variability indicated the positive and negative expression of genes in the population which could not be fixed for heterosis breeding. The heterosis and inbreeding depression jointly implement the criteria for positive selection. As indicated in the present study the succeeding generations showed the significant amount of dominance in the population with accumulation of additive and epistatic genes. Therefore, based of results, it is suggested that the cross combinations should be used for further crop improvement through interest traits to breeder. 


\section{Acknowledgment}

The authors pose sincere thanks to the Department of Genetics and Plant Breeding, Chandra Shekhar Azad University of Agriculture and Technology, Kanpur for providing necessary facilities for carrying out the investigation.

\section{References}

1. Desale CS and DR Mehta. 2013. Heterosis and combining ability analysis for grain yield and quality traits in bread wheat (Triticum aestivum L.). Electronic Journal of Plant Breeding 4(3): 12 05-1213.

2. Deshpandey DP and KA Nayeem. 1999. Heterosis for heat tolerance, protein content, yield and yield components in bread wheat (Triticum aestivum L.). Indian Journal of Genetics and Plant Breeding 59(1): 13-22.

3. Devi EL, Swati, P Goel, M Singh and JP Jaiswal. 2013. Heterosis studies for yield and yield contributing traits in bread wheat (Triticum aestivum L.). The Bioscan 8(3): 905-909.

4. Fonseca S and FL Patterson. 1968.Hybrid vigour in a seven parent diallel cross in common wheat (T. aestivum ). Crop Science 8:85-90.

5. Gaur SC, SN Singh, LP Tiwari and LB Gaur. 2014. Heterosis and inbreeding depression in the inheritance of grain yield and it's components in wheat (Triticum aestivum). Current Advances in Agricultural Sciences 6(2): 186-189.

6. Hassan EE and AMM Saad. 1996. Combining ability, heterosis, correlation and multiple lines regression for yield and its contributing characters in some bread wheat genotypes. Annals of Agricultural Sciences 34(2): 487-499.

7. Jahanzeb F and I Khaliq. 2004. Estimation of heterosis and heterobeltiosis of some quantitative characters in bread wheat crosses. Asian Journal of Plant Sciences 3:508-511.
8. Krystkowiak K, T Admski, M Surma and Z Kaczmarek. 2009. Relationship between phenotypic and genetic diversity of parental genotypes and the specific combining ability and heterosis effects in wheat (Triticum aestivum L.). Euphytica, 165(3): 419-434.

9. Kumar J, SK Singh, L Singh, M Kumar, A Kumar, A Kumar, S Kumar and RK Yadav. 2016. Study of economic heterosis and inbreeding depression in bread wheat (Triticum aestivum L.) under late sown condition. Res. Environ. Life Science 9(9):1082-1086.

10. Meredith WR and RR Bridge. 1972. Heterosis and gene action in cotton Gossypium hirsutum. Crop Science 12: 304-310.

11. Singh M, EL Devi, S Aglawe, N Kousar and C Behera. 2013. Estimation of heterosis in different crosses of bread wheat (Triticum aestivum L.). The Bioscan 8(4): 1393-1401.

12. Singh MK and PK Sharma. 2012. Relationship of heterosis for protein content with yield component trait(s) under normal/heat stress environment in spring wheat. Crop Improvement 39(1): 8-17.

13. Singh MN, PK Sharma, BS Tyagi and G Singh. 2014. Heterosis for yield component traits and protein content in bread wheat under normal and heatstress environment. Cereal Research Communications 42(1): 151-162.

14. Singh P and SS Narayanam. 2009. Biometrical techniques in plant breeding. Fourth Edition, Kalyani Publication, New Delhi. Pp 233.

15. Singh RC. 2003. Effect of heterosis and inbreeding in the inheritance of seed yield and its components in wheat (Triticum aestivum L.). Environment and Ecology 21:903-905. 